\title{
SUBSYMMETRIC WEAK* SCHAUDER BASES AND FACTORIZATION OF THE IDENTITY
}

\author{
RICHARD LECHNER
}

\begin{abstract}
Let $X^{*}$ denote a Banach space with a subsymmetric weak* Schauder basis satisfying condition (C). We show that for any operator $T: X^{*} \rightarrow X^{*}$, either $T\left(X^{*}\right)$ or $\left(\operatorname{Id}_{X^{*}}-T\right)\left(X^{*}\right)$ contains a subspace that is isomorphic to $X^{*}$ and complemented in $X^{*}$. Moreover, we prove that $\ell^{p}\left(X^{*}\right), 1 \leq p \leq \infty$ is primary.
\end{abstract}

\section{INTRODUCTION AND RESULtS}

In [3], Casazza and Lin showed that for any bounded linear projection $Q$ on a Banach space $S$ with a subsymmetric basis, either $Q(S)$ or $(\operatorname{Id}-Q)(S)$ contains a subspace which is isomorphic to $S$ and complemented in $S$. Our first main result Theorem 1.1 extends their result to Banach spaces having a subsymmetric weak* Schauder basis which satisfies the subsequent condition (C).

Condition (C). Let $U$ denote a Banach space with normalized unconditional basis $\left(e_{j}\right)_{j=1}^{\infty}$, whose normalized associated coordinate functionals $\left(e_{j}^{*}\right)_{j=1}^{\infty}$ form a unconditional weak ${ }^{*}$ Schauder basis of $U^{*}$. Given $\mathcal{A} \subset \mathbb{N}$, we define the bounded projection $P_{\mathcal{A}}: U^{*} \rightarrow U^{*}$ by

$$
P_{\mathcal{A}}\left(\sum_{j=1}^{\infty} a_{j} e_{j}^{*}\right)=\sum_{j \in \mathcal{A}}^{\infty} a_{j} e_{j}^{*}
$$

where the above series converge in the weak* topology of $U^{*}$.

We say that $U^{*}$ together with its unconditional weak* Schauder basis $\left(e_{j}^{*}\right)_{j=1}^{\infty}$ satisfies condition $(\mathrm{C})$, if for every infinite set $\Lambda \subset \mathbb{N}$ and every $\theta>0$, we can find a sequence $\left(\mathcal{A}_{j}\right)_{j=1}^{\infty}$ of pairwise disjoint and infinite subsets of $\Lambda$, such that for all $\left(x_{j}^{*}\right)_{j=1}^{\infty} \subset U^{*}$ with $\left\|x_{j}^{*}\right\|_{U^{*}} \leq 1$ there exists a sequence of scalars $\left(a_{j}\right)_{j=1}^{\infty} \in \ell^{1}$ with $\left\|\left(a_{j}\right)_{j=1}^{\infty}\right\|_{\ell^{1}}=1$ such that

$$
\left\|\sum_{j=1}^{\infty} a_{j} P_{\mathcal{A}_{j}} x_{j}^{*}\right\|_{U^{*}} \leq \theta
$$

The above series converges in the weak* topology of $U^{*}$.

Theorem 1.1. Let $S^{*}$ denote a Banach space with a subsymmetric weak* Schauder basis satisfying condition (C). Then for any given bounded operator $T: S^{*} \rightarrow S^{*}$, there exist operators $M, N: S^{*} \rightarrow S^{*}$ such that for $H=T$ or $H=\operatorname{Id}_{S^{*}}-T$, the

Date: July 31, 2018.

2010 Mathematics Subject Classification. 46B25,46B26.

Key words and phrases. Primary, factorization, subsymmetric, weak* Schauder basis.

Supported by the Austrian Science Foundation (FWF) Pr.Nr. P28352. 
diagram

$$
\left.M\right|_{S^{*} \underset{H}{\longrightarrow} S^{*} \stackrel{\operatorname{Id}_{S^{*}}}{\longrightarrow} S^{*}}{ }^{*} \quad\|M\|\|N\| \leq 48 K_{u}^{7} K_{s}^{4}
$$

is commutative. Consequently, $H\left(S^{*}\right)$ contains a subspace which is isomorphic to $S^{*}$ and complemented in $S^{*}$.

We prove Theorem 1.1 in Section 4.

A related concept is the notion of a primary Banach space: a Banach space $X$ is primary, if for every bounded projection $Q: X \rightarrow X$ either $Q(X)$ or $(\operatorname{Id}-Q)(X)$ is isomorphic to $X$ (see e.g. [6]). In [2], Casazza, Kottman and Lin showed that for any Banach space $S$ with a symmetric basis $\left(e_{j}\right)_{j=1}^{\infty}$, the following Banach spaces are primary:

$\triangleright\left(\sum S\right)$, where the direct sum is either the $\ell^{p}$-sum for $1<p<\infty$ or the $c_{0}$-sum, and $S$ is not isomorphic to $\ell^{1}$;

$\triangleright\left(\sum_{n} S_{n}\right)$, where the direct sum is either the $\ell^{p}$-sum for $1<p<\infty$ or the $c_{0}$-sum, and $S_{n}=\operatorname{span}\left\{e_{1}, \ldots, e_{n}\right\}$;

$\triangleright\left(\sum \ell^{\infty}\right)_{p}, 1 \leq p<\infty$.

These results were later complemented by Capon in [1], who showed that the Banach spaces

$\triangleright\left(\sum S\right)_{1}$ and $\left(\sum S\right)_{\infty}$,

$\triangleright\left(\sum_{n} S_{n}\right)_{1}$ and $\left(\sum_{n} S_{n}\right)_{\infty}$

are primary. Theorem 1.2 below adds $\ell^{p}\left(S^{*}\right)$ to that list ( $S^{*}$ may be non-separable).

Theorem 1.2. Let $S^{*}$ denote a Banach space with a subsymmetric weak ${ }^{*}$ Schauder basis satisfying condition (C). Then for all $1 \leq p \leq \infty$ and any given bounded operator $T: \ell^{p}\left(S^{*}\right) \rightarrow \ell^{p}\left(S^{*}\right)$, there exist operators $M, N: \ell^{p}\left(S^{*}\right) \rightarrow \ell^{p}\left(S^{*}\right)$ such that for $H=T$ or $H=\operatorname{Id}_{\ell^{p}\left(S^{*}\right)}-T$, the diagram

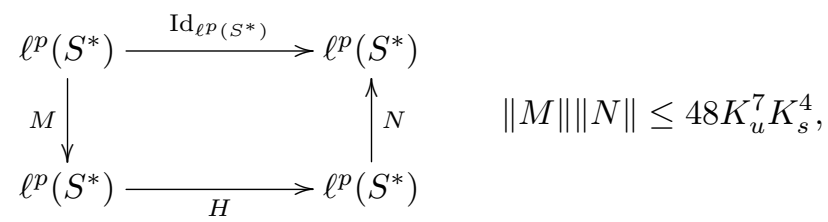

is commutative. Consequently, $\ell^{p}\left(S^{*}\right)$ is primary.

The proof of Theorem 1.2 is given in Section 5 .

The method of proof for both our main theorems is based on the recent result [4].

\section{Notation}

Let $X$ be a Banach space and let $X^{*}$ denote its dual. We say a sequence $\left(x_{n}^{*}\right)_{n=1}^{\infty}$ in $X^{*}$ converges weak $k^{*}$ to $x^{*} \in X^{*}$, if

$$
\lim _{n}\left\langle x_{n}^{*}, x\right\rangle=\left\langle x^{*}, x\right\rangle, \quad x \in X .
$$

In this case we write $\mathrm{w}^{*} \lim x_{n}^{*}=x^{*}$.

We say that $\left(e_{j}^{*}\right)_{j=1}^{\infty} \subset X^{*}$ is a weak Schauder basis for $X^{*}$, if there exists a basis $\left(e_{j}\right)_{j=1}^{\infty}$ for $X$ such that the associated coordinate functionals of $\left(e_{j}\right) j=1^{\infty}$ are $\left(e_{j}^{*}\right)_{j=1}^{\infty}$, i.e. $\left\langle e_{j}^{*}, e_{j}\right\rangle=1$ and $\left\langle e_{j}^{*}, e_{i}\right\rangle=1, i, j \in \mathbb{N}, i \neq j$. Thus,

$$
x^{*}=\mathrm{w}_{n \rightarrow \infty}^{*} \lim _{j=1} \sum^{n}\left\langle x^{*}, e_{j}\right\rangle e_{j}^{*}, \quad x^{*} \in X^{*} .
$$


For more details we refer to $[8,6]$. From now on, whenever we encounter a series in the Banach space

$\triangleright X, U$ or $S$, the mode of convergence is in the norm topology of $X, U$ or $S$;

$\triangleright X^{*}, U^{*}$ or $S^{*}$, the mode of convergence is in the weak* topology of $X^{*}, U^{*}$ or $S^{*}$.

We say the weak ${ }^{*}$ Schauder basis $\left(e_{j}^{*}\right)_{j=1}^{\infty}$ of $X^{*}$ is unconditional, if there exists a constant $C \geq 1$ such that for all sequences of scalars $\left(a_{j}\right)_{j=1}^{\infty}$ with $\sum_{j=1}^{\infty} a_{j} e_{j}^{*} \in X^{*}$ holds that

$$
\left\|\sum_{j=1}^{\infty} \gamma_{j} a_{j} e_{j}^{*}\right\|_{X^{*}} \leq C \sup _{j \in \mathbb{N}}\left|\gamma_{j}\right|\left\|\sum_{j=1}^{\infty} a_{j} e_{j}^{*}\right\|_{X^{*}}, \quad\left(\gamma_{j}\right)_{j=1}^{\infty} \in \ell^{\infty} .
$$

We denote the infimum over all such constants $C \geq 1$ by $K_{u}$. Note that

$$
\left(e_{j}\right)_{j=1}^{\infty} \text { is unconditional if and only if } \quad\left(e_{j}^{*}\right)_{j=1}^{\infty} \text { is unconditional. }
$$

We say that a weak* Schauder basis $\left(e_{j}^{*}\right)_{j=1}^{\infty}$ of $X^{*}$ is subsymmetric, if the following two conditions are satisfied:

(a) $\left(e_{j}^{*}\right)_{j=1}^{\infty}$ is unconditional;

(b) Let $\left(n_{j}\right)_{j=1}^{\infty} \subset \mathbb{N}$ be an increasing sequence, and let $Y_{\left(n_{j}\right)_{j=1}^{\infty}}$ denote the weak* closure of $\operatorname{span}\left\{e_{n_{j}}^{*}\right\}_{j=1}^{\infty}$. Then the operator $S_{\left(n_{j}\right)_{j=1}^{\infty}}: X^{*} \rightarrow Y_{\left(n_{j}\right)_{j=1}^{\infty}}$ given by

$$
S_{\left(n_{j}\right)_{j=1}^{\infty}}\left(\sum_{j=1}^{\infty} a_{j} e_{j}^{*}\right)=\sum_{j=1}^{\infty} a_{j} e_{n_{j}}^{*}
$$

is isomorphic.

Note that

$$
\left(e_{j}\right)_{j=1}^{\infty} \text { is subsymmetric } \quad \text { if and only if } \quad\left(e_{j}^{*}\right)_{j=1}^{\infty} \text { is subsymmetric. }
$$

We define $K_{s}$ by

$$
K_{s}=\sup \left\{\left\|S_{\left(n_{j}\right)_{j=1}^{\infty}}\right\|,\left\|S_{\left(n_{j}\right)_{j=1}^{\infty}}^{-1}\right\|:\left(n_{j}\right)_{j=1}^{\infty} \subset \mathbb{N} \text { is increasing }\right\} .
$$

The constant $K_{s}$ is finite (see [8, Chapter II, Theorem 21.2]).

For all $1 \leq p \leq \infty$ and for any Banach space $X$, we define as usual $\ell^{p}(X)$ by

$$
\left\{\left(y_{n}\right)_{n=1}^{\infty}: y_{n} \in X \text { and }\left\|\left(y_{n}\right)_{n=1}^{\infty}\right\|_{\ell^{p}(X)}=\left(\sum_{n=1}^{\infty}\left\|y_{n}\right\|_{X}\right)^{1 / p}<\infty\right\} .
$$

We conclude this section with two remarks on condition (C).

Remark 2.1. Any Banach space $U^{*}$ with an unconditional weak* Schauder basis $\left(e_{j}^{*}\right)$ in which $\ell^{1}$ does not embed, satisfies condition $(\mathrm{C})$.

Remark 2.2. $\ell^{\infty}$ with the standard unit vector weak* Schauder basis $\left(e_{j}\right)_{j=1}^{\infty}$ satisfies condition (C).

First, we will show that Remark 2.1 is indeed correct. To this end, assume that condition (C) does not hold. Then there exists an infinite set $\Lambda \subset \mathbb{N}$ and a $\theta>0$, such that for any sequence $\left(\mathcal{A}_{j}\right)_{j=1}^{\infty}$ of pairwise disjoint and infinite subsets of $\Lambda$, we can find $\left(x_{j}^{*}\right)_{j=1}^{\infty} \subset U^{*}$ with $\left\|x_{j}^{*}\right\|_{U^{*}} \leq 1$ such that for all scalars $\left(a_{j}\right)_{j=1}^{\infty} \in \ell^{1}$ with $\left\|\left(a_{j}\right)_{j=1}^{\infty}\right\|_{\ell^{1}}=1$ we have

$$
\left\|\sum_{j=1}^{\infty} a_{j} P_{\mathcal{A}_{j}} x_{j}^{*}\right\|_{U^{*}}>\theta
$$


If we put $y_{j}^{*}=P_{\mathcal{A}_{j}} x_{j}^{*}$ and $\widetilde{y}_{j}^{*}=y_{j}^{*} /\left\|y_{j}^{*}\right\|_{U^{*}}, j \in \mathbb{N}$, then we obtain by unconditionality

$$
\left\|\sum_{j=1}^{\infty} a_{j} \widetilde{y}_{j}^{*}\right\|_{U^{*}}>\theta / K_{u}
$$

thus, $\left(\widetilde{y}_{j}^{*}\right)_{j=1}^{\infty}$ is equivalent to the standard unit vector basis of $\ell^{1}$.

To see that Remark 2.2 is valid, let $\Lambda \subset \mathbb{N}$ denote an infinite set, and let $\theta>0$. Pick any sequence $\left(\mathcal{A}_{j}\right)_{j=1}^{\infty}$ of pairwise disjoint subsets of $\Lambda$, and let $\left(x_{j}\right)_{j=1}^{\infty}$ denote any sequence of vectors in $\ell^{\infty}$ with $\left\|x_{j}\right\|_{\ell^{\infty}} \leq 1, j \in \mathbb{N}$. Choose $N \in \mathbb{N}$ such that $\frac{1}{N} \leq \theta$, and define $a_{j}=\frac{1}{N}$ if $j \leq N$, and $a_{j}=0$ whenever $j>N$. Then clearly

$$
\left\|\sum_{j=1}^{\infty} a_{j} P_{\mathcal{A}_{j}} x_{j}\right\|_{\ell^{\infty}}=\frac{1}{N} \leq \theta .
$$

\section{Two SUBSPACE ANNIHILATION LEMMATA}

We will present two lemmata, which are used in the proof of our first main result Theorem 1.1 to almost diagonalize an operator $T: X^{*} \rightarrow X^{*}$. Although we use Lemma 3.1 only for $m=1$ here, we give the proof for general $m \in \mathbb{N}$ here, for later reference.

Lemma 3.1. Let $X$ denote a Banach space with a normalized basis $\left(e_{j}\right)_{j=1}^{\infty}$, such that the associated coordinate functionals $\left(e_{j}^{*}\right)_{j=1}^{\infty}$ form a weak $k^{*}$ Schauder basis of $X^{*}$. Let $\Lambda_{0} \subset \mathbb{N}$ denote an infinite set, $m, n \in \mathbb{N}, x_{1}^{*}, \ldots, x_{n}^{*} \in X^{*}$ and $\eta>0$. Then we can find a finite set $\mathcal{F} \subset \Lambda_{0}$ with cardinality $|\mathcal{F}|=2 m$ such that

$$
\max _{1 \leq j \leq n}\left|\left\langle\sum_{k \in \mathcal{F}} \varepsilon_{k} e_{k}, x_{j}^{*}\right\rangle\right| \leq \eta, \quad \varepsilon \in \mathcal{E},
$$

where the set of signs $\mathcal{E}=\mathcal{E}(\mathcal{F})$ is given by

$$
\mathcal{E}=\left\{\left(\varepsilon_{k}\right)_{k \in \mathcal{F}}: \varepsilon_{k} \in\{ \pm 1\}, \sum_{k \in \mathcal{F}} \varepsilon_{k}=0\right\} .
$$

Proof. Firstly, we will prove that given $x^{*} \in X^{*}$, there exists an infinite set $\Lambda \subset \Lambda_{0}$ such that

$$
\left|\left\langle e_{k}, x^{*}\right\rangle-\left\langle e_{k^{\prime}}, x^{*}\right\rangle\right| \leq \frac{\eta}{m}, \quad k, k^{\prime} \in \Lambda .
$$

To this end, we define the sets

$$
\mathcal{M}_{\ell}=\left\{j \in \Lambda_{0}: \frac{\eta}{2 m}(\ell-1) \leq\left\langle e_{j}, x^{*}\right\rangle<\frac{\eta}{2 m} \ell\right\}, \quad \ell \in \mathbb{Z} .
$$

Since $\left|\left\langle e_{j}, x^{*}\right\rangle\right| \leq\left\|x^{*}\right\|<\infty$, there are only finitely many $\ell \in \mathbb{Z}$ for which $\mathcal{M}_{\ell}$ is non-empty. Hence, since $\bigcup_{\ell \in \mathbb{Z}} \mathcal{M}_{l}=\Lambda_{0}$, at least one of the sets $\mathcal{M}_{\ell}, \ell \in \mathbb{Z}$ has to be infinite. Clearly, this infinite set satisfies (3.1).

Now we will just repeatedly use (3.1). We begin by selecting $\Lambda_{1} \subset \Lambda_{0}$ so that

$$
\left|\left\langle e_{k}, x_{1}^{*}\right\rangle-\left\langle e_{k^{\prime}}, x_{1}^{*}\right\rangle\right| \leq \frac{\eta}{m}, \quad k, k^{\prime} \in \Lambda_{1} .
$$

Let $1 \leq i \leq n-1$ and assume we have already chosen infinite sets $\Lambda_{0} \supset \Lambda_{1} \supset \Lambda_{2} \supset$ $\cdots \supset \Lambda_{i-1}$ such that

$$
\max _{1 \leq j \leq i-1}\left|\left\langle e_{k}, x_{j}^{*}\right\rangle-\left\langle e_{k^{\prime}}, x_{j}^{*}\right\rangle\right| \leq \frac{\eta}{m}, \quad k, k^{\prime} \in \Lambda_{i-1} .
$$

By replacing $\Lambda_{0}$ with the infinite set $\Lambda_{i-1}$ in (3.1), we can find an infinite set $\Lambda_{i} \subset \Lambda_{i-1}$ such that

$$
\max _{1 \leq j \leq i}\left|\left\langle e_{k}, x_{j}^{*}\right\rangle-\left\langle e_{k^{\prime}}, x_{j}^{*}\right\rangle\right| \leq \frac{\eta}{m}, \quad k, k^{\prime} \in \Lambda_{i}
$$


Stopping the induction after $n$ steps, we obtain

$$
\max _{1 \leq j \leq n}\left|\left\langle e_{k}, x_{j}^{*}\right\rangle-\left\langle e_{k^{\prime}}, x_{j}^{*}\right\rangle\right| \leq \frac{\eta}{m}, \quad k, k^{\prime} \in \Lambda_{n}
$$

We choose any $\mathcal{F} \subset \Lambda_{n}$ with $|\mathcal{F}|=2 m$ and let $\varepsilon=\left(\varepsilon_{k}\right)_{k \in \mathcal{F}} \in \mathcal{E}=\mathcal{E}(\mathcal{F})$, i.e. $\varepsilon_{k} \in\{ \pm 1\}$ is such that $\sum_{k \in \mathcal{F}} \varepsilon_{k}=0$. Then since $\mathcal{F} \subset \Lambda_{n}$, we obtain from (3.2) that

$$
\left|\left\langle\sum_{k \in \mathcal{F}} \varepsilon_{k} e_{k}, x_{j}^{*}\right\rangle\right|=\left|\sum_{\substack{k \in \mathcal{F} \\ \varepsilon_{k}=1}}\left\langle e_{k}, x_{j}^{*}\right\rangle-\sum_{\substack{k \in \mathcal{F} \\ \varepsilon_{k}=-1}}\left\langle e_{k}, x_{j}^{*}\right\rangle\right| \leq \eta, \quad 1 \leq j \leq n .
$$

The following Lemma is an abstract version of an argument which Lindenstrauss used in [5] (see also [6]) to show that $\ell^{\infty}$ is prime (which means that every infinite dimensional complemented subspace of $\ell^{\infty}$ is isomorphic to $\left.\ell^{\infty}\right)$.

Lemma 3.2. Let $U$ denote a Banach space with a normalized unconditional basis $\left(e_{j}\right)_{j=1}^{\infty}$, such that the associated coordinate functionals $\left(e_{j}^{*}\right)_{j=1}^{\infty}$ form an unconditional weak $k^{*}$ Schauder basis of $U^{*}$. Let $\Lambda \subset \mathbb{N}$ denote an infinite set, $\eta>0$ and $\varphi \in U^{* *}$. If $U^{*}$ together with $\left(e_{j}^{*}\right)_{j=1}^{\infty}$ satisfies condition $(\mathrm{C})$, then there exists an infinite set $\mathcal{A} \subset \Lambda$ such that

$$
\sup _{\left\|x^{*}\right\|_{U^{*}} \leq 1}\left|\left\langle\varphi, P_{\mathcal{A}} x^{*}\right\rangle\right| \leq \eta
$$

Proof. Let $\eta>0, \varphi \in U^{* *} \backslash\{0\}$, and assume the conclusion of the Lemma is false. Hence, for all infinite sets $\mathcal{A} \subset \Lambda$ there exists $x^{*}$ with $\left\|x^{*}\right\|_{U^{*}}=1$ such that

$$
\left\langle\varphi, P_{\mathcal{A}} x^{*}\right\rangle>\eta
$$

We define $\theta=\frac{\eta}{2\|\varphi\|_{U^{* *} K_{u}}}$ and choose $\left(\mathcal{A}_{j}\right)_{j=1}^{\infty}$ with $\mathcal{A}_{j} \subset \Lambda, j \in \mathbb{N}$ according to condition (C). By our assumption (3.3), we can find $x_{j}^{*} \in U^{*}$ with $\left\|x_{j}^{*}\right\|_{U^{*}}=1$, $j \in \mathbb{N}$, such that

$$
\left\langle\varphi, P_{\mathcal{A}_{j}} x_{j}^{*}\right\rangle>\eta
$$

By condition (C) we can find a sequence $\left(a_{j}\right)_{j=1}^{\infty} \in \ell^{1}$ with $\left\|\left(a_{j}\right)_{j=1}^{\infty}\right\|_{\ell^{1}}=1$ such that

$$
\left\|\sum_{j=1}^{\infty} a_{j} P_{\mathcal{A}_{j}} x_{j}^{*}\right\|_{U^{*}} \leq \theta=\frac{\eta}{2\|\varphi\|_{U^{* *}} K_{u}} .
$$

But on the other hand, we obtain from (3.4) that

$$
\left\langle\varphi, \sum_{j=1}^{n} a_{j} P_{\mathcal{A}_{j}} x_{j}^{*}\right\rangle>\eta
$$

for a large enough $n \in \mathbb{N}$. Combining the latter estimate with (3.5), we arrive at the contradiction

$$
\eta<\left\langle\varphi, \sum_{j=1}^{n} a_{j} P_{\mathcal{A}_{j}} x_{j}^{*}\right\rangle \leq\|\varphi\|_{U^{* *}}\left\|\sum_{j=1}^{n} a_{j} P_{\mathcal{A}_{j}} x_{j}^{*}\right\|_{U^{*}} \leq \eta / 2 .
$$

\section{Proof of Theorem 1.1}

For convenience of the reader, we restate Theorem 1.1 here below.

Theorem 4.1 (Main result Theorem 1.1). Let $S^{*}$ denote a Banach space with a subsymmetric weak* Schauder basis satisfying condition (C). Then for any given 
bounded operator $T: S^{*} \rightarrow S^{*}$, there exist operators $M, N: S^{*} \rightarrow S^{*}$ such that for $H=T$ or $H=\operatorname{Id}_{S^{*}}-T$, the diagram

$$
\left.M\right|_{S^{*} \underset{H}{\longrightarrow} S^{*} \stackrel{\operatorname{Id}_{S^{*}}}{\longrightarrow} S^{*}} ^{S^{*}} \quad\|M\|\|N\| \leq 48 K_{u}^{7} K_{s}^{4}
$$

is commutative. Consequently, $H\left(S^{*}\right)$ contains a subspace which is isomorphic to $S^{*}$ and complemented in $S^{*}$.

Proof. In this proof, we use the following constants:

$$
\eta_{i}=K_{u}^{-1} 4^{-i-1}, \quad i \in \mathbb{N} .
$$

Step 1: Inductive COnstruction of The Block BAsis. Let $\left(s_{j}\right)_{j=1}^{\infty}$ denote the subsymmetric basis of $S$, and let $\left(s_{j}^{*}\right)_{j=1}^{\infty}$ denote the associated coordinate functionals, which form a weak* Schauder basis for $S^{*}$, such that condition (C) is satisfied. We put $\mathcal{A}_{1}=\mathbb{N}$, choose $\mathcal{B}_{1}=\{1,2\}$, and we define

$$
b_{1}=s_{1}-s_{2} \quad \text { and } \quad b_{1}^{*}=s_{1}^{*}-s_{2}^{*} .
$$

By Lemma 3.2, there exists an infinite collection $\mathcal{A}_{2} \subset \mathcal{A}_{1} \backslash\{1,2\}$, such that

$$
\sup _{\left\|x^{*}\right\|_{S^{*}} \leq 1}\left|\left\langle T^{*} b_{1}, P_{\mathcal{A}_{2}} x^{*}\right\rangle\right| \leq \eta_{1} .
$$

Now assume we have already chosen infinite sets $\mathcal{A}_{1} \supset \mathcal{A}_{2} \supset \cdots \supset \mathcal{A}_{i}$, pairwise disjoint finite sets $\mathcal{B}_{j} \subset \mathcal{A}_{j}$ with $\left|\mathcal{B}_{j}\right|=2,1 \leq j \leq i-1$ and that we have defined

$$
b_{j}=s_{k_{0}}-s_{k_{1}} \quad \text { and } \quad b_{j}^{*}=s_{k_{0}}^{*}-s_{k_{1}}^{*}, \quad k_{0}, k_{1} \in \mathcal{B}_{j}, k_{0}<k_{1},
$$

for all $1 \leq j \leq i-1$. By Lemma 3.1, we can find a set $\mathcal{B}_{i} \subset \mathcal{A}_{i}$ with $\left|\mathcal{B}_{i}\right|=2$ so that if we put

$$
b_{i}=s_{k_{0}}-s_{k_{1}} \quad \text { and } \quad b_{i}^{*}=s_{k_{0}}^{*}-s_{k_{1}}^{*}, \quad k_{0}, k_{1} \in \mathcal{B}_{i}, k_{0}<k_{1},
$$

we have the estimate

$$
\sum_{j=1}^{i-1}\left|\left\langle b_{i}, T b_{j}^{*}\right\rangle\right| \leq \eta_{i}
$$

By Lemma 3.2, there exists an infinite set $\mathcal{A}_{i+1}$ with

$$
\mathcal{A}_{i+1} \subset \mathcal{A}_{i} \backslash\left\{k \in \mathbb{N}: k \leq \max \left(\bigcup_{j=1}^{i} \mathcal{B}_{i}\right)\right\}
$$

such that

$$
\sup _{\left\|x^{*}\right\|_{S^{*}} \leq 1}\left|\left\langle T^{*} b_{i}, P_{\mathcal{A}_{i+1}} x^{*}\right\rangle\right| \leq \eta_{i} .
$$

This completes the inductive step.

The estimates (4.4), (4.7) and (4.9) imply

$$
\begin{array}{cc}
\sum_{j=1}^{i-1}\left|\left\langle b_{i}, T b_{j}^{*}\right\rangle\right| \leq \eta_{i}, & i \in \mathbb{N}, \\
\left|\left\langle b_{i}, T \sum_{j=i+1}^{\infty} a_{j} b_{j}^{*}\right\rangle\right| \leq \eta_{i}\left\|\sum_{j=i+1}^{\infty} a_{j} b_{j}^{*}\right\|_{S^{*}}, & i \in \mathbb{N},
\end{array}
$$


SUBSYMMETRIC WEAK* SCHAUDER BASES AND FACTORIZATION OF THE IDENTITY 7 whenever $\left(a_{j}\right)_{j=1}^{\infty} \subset \mathbb{R}$ is such that $\sum_{j=i+1}^{\infty} a_{j} b_{j}^{*} \in S^{*}$.

STEP 2: BAsic operators. We define the operators $B, Q: S^{*} \rightarrow S^{*}$ by

$$
\begin{array}{rlrl}
B x^{*} & =\sum_{j=1}^{\infty} \frac{1}{\left\|b_{j}^{*}\right\|_{S^{*}}}\left\langle x^{*}, s_{j}\right\rangle b_{j}^{*}, & x^{*} \in S^{*}, \\
Q x^{*}=\sum_{j=1}^{\infty} \frac{\left\|b_{j}^{*}\right\|_{S^{*}}}{\left|\mathcal{B}_{j}\right|}\left\langle b_{j}, x^{*}\right\rangle s_{j}^{*}, & x^{*} \in S^{*} .
\end{array}
$$

We will now show that $B$ and $Q$ are bounded linear operators. To this end, let $x^{*}=\sum_{j=1}^{\infty} a_{j} s_{j}^{*}$. Firstly, note that

$$
\left\|B\left(\sum_{j=1}^{\infty} a_{j} s_{j}^{*}\right)\right\|_{S^{*}} \leq\left\|\sum_{j=1}^{\infty} \frac{1}{\left\|b_{j}^{*}\right\|_{S^{*}}} a_{j} s_{k_{0}(j)}^{*}\right\|_{S^{*}}+\left\|\sum_{j=1}^{\infty} \frac{1}{\left\|b_{j}^{*}\right\|_{S^{*}}} a_{j} s_{k_{1}(j)}^{*}\right\|_{S^{*}},
$$

where $\left\{k_{0}(j), k_{1}(j)\right\}=\mathcal{B}_{j}, j \in \mathbb{N}$. By unconditionality, we obtain $\left\|b_{j}^{*}\right\|_{S^{*}} \geq K_{u}^{-1}$ and

$$
\left\|B\left(\sum_{j=1}^{\infty} a_{j} s_{j}^{*}\right)\right\|_{S^{*}} \leq K_{u}^{2}\left(\left\|\sum_{j=1}^{\infty} a_{j} s_{k_{0}(j)}^{*}\right\|_{S^{*}}+\left\|\sum_{j=1}^{\infty} a_{j} s_{k_{1}(j)}^{*}\right\|_{S^{*}}\right),
$$

Furthermore, by (4.8) we have that $s_{k_{0}(j)}<s_{k_{0}(j+1)}$ and $s_{k_{1}(j)}<s_{k_{1}(j+1)}, j \in \mathbb{N}$. The weak* Schauder basis $\left(s_{j}^{*}\right)_{j=1}^{\infty}$ is subsymmetric, hence

$$
\left\|B\left(\sum_{j=1}^{\infty} a_{j} s_{j}^{*}\right)\right\|_{S^{*}} \leq 2 K_{u}^{2} K_{s}\left\|\sum_{j=1}^{\infty} a_{j} s_{j}^{*}\right\|_{S^{*}} .
$$

Secondly, if we write $b_{j}=s_{k_{0}}(j)-s_{k_{1}}(j)$ for $k_{0}, k_{1} \in \mathcal{B}_{j}, k_{0}<k_{1}, j \in \mathbb{N}$, we obtain

$$
\left\|Q x^{*}\right\|_{S^{*}} \leq\left\|\sum_{j=1}^{\infty} \frac{\left\|b_{j}^{*}\right\|_{S^{*}}}{\left|\mathcal{B}_{j}\right|}\left\langle s_{k_{0}(j)}, x^{*}\right\rangle s_{j}^{*}\right\|_{S^{*}}+\left\|\sum_{j=1}^{\infty} \frac{\left\|b_{j}^{*}\right\|_{S^{*}}}{\left|\mathcal{B}_{j}\right|}\left\langle s_{k_{1}(j)}, x^{*}\right\rangle s_{j}^{*}\right\|_{S^{*}} .
$$

Since $\left(s_{j}^{*}\right)_{j=1}^{\infty}$ is subsymmetric, the right hand side of the latter inequality is dominated by

$$
K_{s}\left\|\sum_{j=1}^{\infty} \frac{\left\|b_{j}^{*}\right\|_{S^{*}}}{\left|\mathcal{B}_{j}\right|}\left\langle s_{k_{0}(j)}, x^{*}\right\rangle s_{k_{0}(j)}^{*}\right\|_{S^{*}}+K_{s}\left\|\sum_{j=1}^{\infty} \frac{\left\|b_{j}^{*}\right\|_{S^{*}}}{\left|\mathcal{B}_{j}\right|}\left\langle s_{k_{1}(j)}, x^{*}\right\rangle s_{k_{1}(j)}^{*}\right\|_{S^{*}} .
$$

Since $\frac{\left\|b_{j}^{*}\right\|_{S^{*}}}{\left|\mathcal{B}_{j}\right|} \leq 1$, unconditionality yields

$$
\left\|Q x^{*}\right\|_{S^{*}} \leq 2 K_{s} K_{u}\left\|x^{*}\right\|_{S^{*}} .
$$

One can easily verify that $Q B=\operatorname{Id}_{S^{*}}$, i.e. the diagram

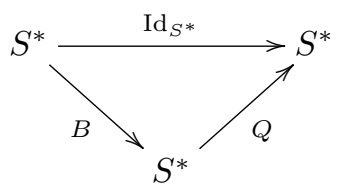

is commutative. Consequently, $B$ is an isomorphism onto its range and its range is complemented by $B Q$.

Step 3: Conclusion of The Proof. Observe that at least one of the two following sets is infinite:

$$
\left\{j \in \mathbb{N}:\left|\left\langle b_{j}, T b_{j}^{*}\right\rangle\right| \geq 1\right\} \quad \text { or } \quad\left\{j \in \mathbb{N}:\left|\left\langle b_{j},\left(\operatorname{Id}_{S^{*}}-T\right) b_{j}^{*}\right\rangle\right| \geq 1\right\} .
$$


If the left set is infinite we denote it by $\mathcal{J}$ and put $H=T$, and if the left set is finite, we denote the right set by $\mathcal{J}$ and we define $H=\operatorname{Id}_{S^{*}}-T$. In either case, we obtain

$$
\mathcal{J} \text { is infinite } \quad \text { and } \quad\left|\left\langle b_{j}, H b_{j}^{*}\right\rangle\right| \geq 1, \quad j \in \mathcal{J} .
$$

Let $Y=B\left(S^{*}\right)$ and note that (4.12), (4.13) and (4.14) yields

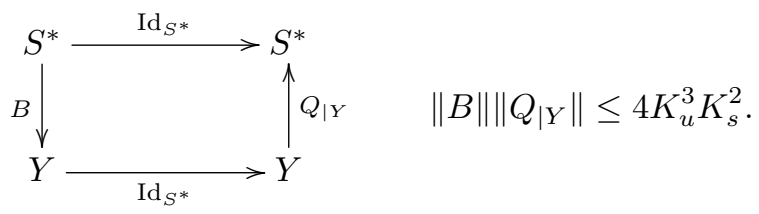

Now, define $P: S^{*} \rightarrow Y$ by

$$
P x^{*}=\sum_{j \in \mathcal{J}} \frac{\left\langle b_{j}, x^{*}\right\rangle}{\left\langle b_{j}, H b_{j}^{*}\right\rangle} b_{j}^{*}, \quad x^{*} \in S^{*},
$$

and observe that by $(4.15)$, the unconditionality of $\left(s_{j}^{*}\right)_{j=1}^{\infty}$ (and thus, the unconditionality of $\left.\left(b_{j}^{*}\right)_{j=1}^{\infty}\right)$ and the definition of $B$ and $Q$ (see (4.11)), we obtain

$$
\left\|P x^{*}\right\|_{S^{*}} \leq 2 K_{u}\left\|B Q x^{*}\right\|_{S^{*}}, \quad x^{*} \in S^{*} .
$$

Combining the latter estimate with (4.12) and (4.13) yields

$$
\left\|P x^{*}\right\|_{S^{*}} \leq 8 K_{u}^{4} K_{s}^{2}\left\|x^{*}\right\|_{S^{*}}, \quad x^{*} \in S^{*} .
$$

A straightforward calculation shows that for all $y=\sum_{j \in \mathcal{J}} a_{j} b_{j}^{*} \in Y$, the following identity is true:

$$
P H y-y=\sum_{i \in \mathcal{J}} \sum_{j \in \mathcal{J}: j<i} a_{j} \frac{\left\langle b_{i}, H b_{j}^{*}\right\rangle}{\left\langle b_{i}, H b_{i}^{*}\right\rangle} b_{i}^{*}+\sum_{i \in \mathcal{J}} \frac{\left\langle b_{i}, H \sum_{j \in \mathcal{J}: j>i} a_{j} b_{j}^{*}\right\rangle}{\left\langle b_{i}, H b_{i}^{*}\right\rangle} b_{i}^{*} .
$$

Since $\left|\left\langle b_{j}, y\right\rangle\right| \leq\left\|b_{j}\right\|_{S}\|y\|_{S^{*}}$, we obtain $\left|a_{j}\right| \leq\|y\|_{S^{*}}$. Hence, by (4.15) and the crucial off diagonal estimate (4.10a), we obtain

$$
\left\|\sum_{i \in \mathcal{J}} \sum_{j \in \mathcal{J}: j<i} a_{j} \frac{\left\langle b_{i}, H b_{j}^{*}\right\rangle}{\left\langle b_{i}, H b_{i}^{*}\right\rangle} b_{i}^{*}\right\|_{S^{*}} \leq 2\|y\|_{S^{*}} \sum_{i \in \mathbb{N}} \eta_{i}
$$

Using (4.15) and the second off diagonal estimate $(4.10 \mathrm{~b})$, we obtain by unconditionality

$$
\begin{aligned}
\left\|\sum_{i \in \mathcal{J}} \frac{\left\langle b_{i}, H \sum_{j \in \mathcal{J}: j>i} a_{j} b_{j}^{*}\right\rangle}{\left\langle b_{i}, H b_{i}^{*}\right\rangle} b_{i}^{*}\right\|_{S^{*}} & \leq 2 \sum_{i \in \mathcal{J}} \eta_{i}\left\|\sum_{j \in \mathcal{J}: j>i} a_{j} b_{j}^{*}\right\|_{S^{*}} \\
& \leq 2 K_{u}\|y\|_{S^{*}} \sum_{i \in \mathbb{N}} \eta_{i} .
\end{aligned}
$$

Recall that in $(4.2)$ we put $\eta_{i}=K_{u}^{-1} 4^{-i-1}, i \in \mathbb{N}$. Thus, inserting the estimates (4.20) and (4.21) into (4.19) yields

$$
\|P H y-y\|_{S^{*}} \leq \frac{1}{3}\|y\|_{S^{*}}, \quad y \in Y .
$$

Let $J: Y \rightarrow S^{*}$ denote the operator given by $J y=y$, then, by (4.22), PHJ : $Y \rightarrow Y$ is invertible and $\left\|(P H J)^{-1}\right\| \leq \frac{3}{2}$. Thus, if we define $V=(P H J)^{-1} P$, the 
diagram

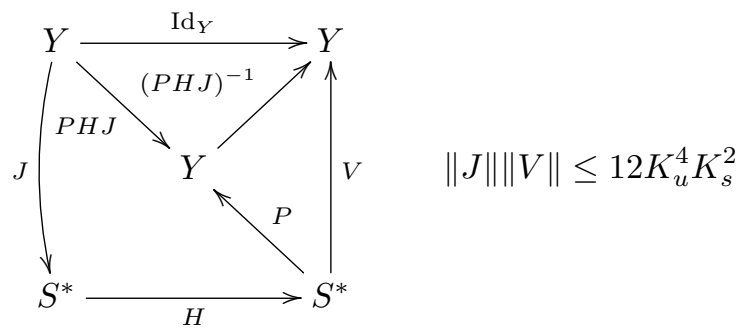

is commutative. Merging the diagrams (4.16) and (4.23) concludes the proof:

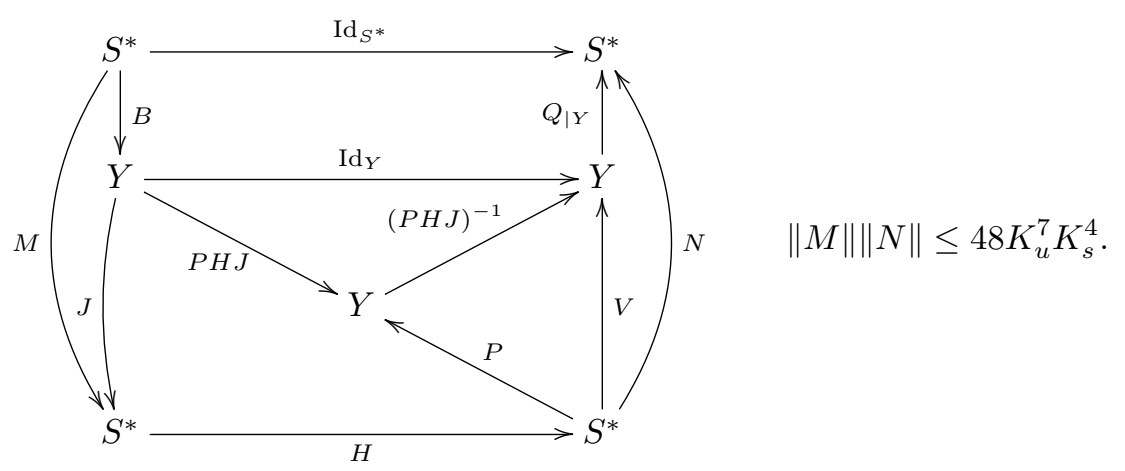

\section{Direct sums of BANACH SPACES With A WEAK* SUbSYMmetriC Basis}

We begin this section by introducing notation specific to the two parameter case. Then we extend the subspace annihilation lemmata in Section 3 to the two parameter case and use them to prove our second main result Theorem 1.2.

5.1. Notation. Let $X$ denote a Banach space with normalized basis $\left(x_{j}\right)_{j=1}^{\infty}$, such that their normalized associated coordinate functionals $\left(x_{j}^{*}\right)_{j=1}^{\infty}$ form a weak* Schauder basis of $X^{*}$. For each $i \in \mathbb{N}$, let $q_{i}: \ell^{p}\left(X^{*}\right) \rightarrow X^{*}$ denote the canonical norm 1 coordinate projection given by

$$
y_{i}\left(\left(y_{n}\right)_{n=1}^{\infty}\right)=y_{i}, \quad\left(y_{n}\right)_{n=1}^{\infty} \in \ell^{p}\left(X^{*}\right) .
$$

Let $\mathcal{J} \subset \mathbb{N}$ and define $Q_{\mathcal{J}}: \ell^{p}\left(X^{*}\right) \rightarrow \ell^{p}\left(X^{*}\right)$ as the natural norm 1 projection onto the coordinates indexed by $\mathcal{J}$, i.e.

$$
q_{i} Q_{\mathcal{J}}\left(y_{n}\right)_{n=1}^{\infty}=y_{i}, i \in \mathcal{J} \quad \text { and } \quad q_{i} Q_{\mathcal{J}}\left(y_{n}\right)_{n=1}^{\infty}=0, i \notin \mathcal{J},
$$

for all $\left(y_{n}\right)_{n=1}^{\infty} \in \ell^{p}\left(X^{*}\right)$. For $i \in \mathbb{N}$, we will sometimes write $Q_{i}$ instead of $Q_{\{i\}}$. For each $i \in \mathbb{N}$, we define $I_{i}: X^{*} \rightarrow \ell^{p}\left(X^{*}\right)$ as the canonical isometric embedding of $X^{*}$ into the $i$-th coordinate of $\ell^{p}\left(X^{*}\right)$, which is given by

$$
q_{i} I_{i} x^{*}=x^{*} \quad \text { and } \quad q_{k} I_{i} x^{*}=0, \quad k \in \mathbb{N}, k \neq i, \quad i \in \mathbb{N} .
$$

We define the sequence $\left(e_{i j}\right)_{i, j=1}^{\infty} \subset \ell^{p}\left(X^{*}\right)$ by

$$
e_{i j}=I_{i} x_{j}^{*}, \quad i, j \in \mathbb{N}
$$

hence, the associated coordinate functionals $f_{i j}: \ell^{p}\left(X^{*}\right) \rightarrow \mathbb{R}$ are given by

$$
\left\langle f_{i j}, y\right\rangle=\left\langle q_{i} y, x_{j}\right\rangle, \quad y \in \ell^{p}\left(X^{*}\right), i, j \in \mathbb{N} .
$$

Given any sequence of scalars $\left(a_{i j}\right)_{i, j=1}^{\infty}$ for which $\left(\mathrm{w}^{*} \lim _{n \rightarrow \infty} \sum_{j=1}^{n} a_{i j} x_{j}^{*}\right)_{i=1}^{\infty} \in$ $\ell^{p}\left(X^{*}\right)$, we define

$$
\sum_{i, j=1}^{\infty} a_{i j} e_{i j}=\left(\sum_{j=1}^{\infty} a_{i j} x_{j}^{*}\right)_{i=1}^{\infty}=\left(\mathrm{w}_{n \rightarrow \infty}^{*} \lim _{j=1} \sum_{i j}^{n} a_{j}^{*}\right)_{i=1}^{\infty} .
$$


Thus, we have the identity

$$
\sum_{i, j=1}^{\infty}\left\langle f_{i j}, y\right\rangle e_{i j}=\left(\sum_{j=1}^{\infty}\left\langle q_{i} y, x_{j}\right\rangle x_{j}^{*}\right)_{i=1}^{\infty}=y, \quad y \in \ell^{p}\left(X^{*}\right) .
$$

For $\mathcal{K} \subset \mathbb{N}^{2}$, we define the projection $R_{\mathcal{K}}: \ell^{p}\left(X^{*}\right) \rightarrow \ell^{p}\left(X^{*}\right)$ by

$$
R_{\mathcal{K}} y=\sum_{i, j=1}^{\infty}\left\langle f_{i j}, y\right\rangle \mathbb{1}_{\mathcal{K}}(i, j) e_{i j}
$$

where $\mathbb{1}_{\mathcal{K}}(i, j)=1$ if $(i, j) \in \mathcal{K}$, and $\mathbb{1}_{\mathcal{K}}(i, j)=0$ if $(i, j) \notin \mathcal{K}$. If $\left(x_{j}^{*}\right)$ is unconditional, then $R_{\mathcal{K}}$ is bounded.

5.2. Two subspace annihilation lemmata for direct sums. The following lemmata are two parameter versions of the subspace annihilation lemmata presented in Section 3.

Lemma 5.1. Let $X$ denote a Banach space with a normalized basis $\left(x_{j}\right)_{j=1}^{\infty}$, such that their normalized associated coordinate functionals $\left(x_{j}^{*}\right)_{j=1}^{\infty}$ form a weak* Schauder basis of $X^{*}$. Let $1 \leq p \leq \infty$ and let $\Lambda_{0} \subset \mathbb{N}$ denote an infinite set. Then, given $m \in \mathbb{N}, y_{1}, \ldots, y_{m} \in \ell^{p}\left(X^{*}\right), n_{0} \in \mathbb{N}, \eta>0$ and $M \in \mathbb{N}$, there exists a finite set $\mathcal{F} \subset \Lambda_{0}$ with $|\mathcal{F}|=2 M$ such that

$$
\max _{1 \leq \ell \leq m}\left|\left\langle\sum_{k \in \mathcal{F}} \varepsilon_{n_{0} k} f_{n_{0} k}, y_{\ell}\right\rangle\right| \leq \eta, \quad \varepsilon \in \mathcal{E},
$$

where the set of signs $\mathcal{E}=\mathcal{E}\left(n_{0}, \mathcal{F}\right)$ is given by

$$
\mathcal{E}=\left\{\left(\varepsilon_{n_{0} k}\right)_{k \in \mathcal{F}}: \varepsilon_{n_{0} k} \in\{ \pm 1\}, \sum_{k \in \mathcal{F}} \varepsilon_{n_{0} k}=0\right\}
$$

Proof. Since the proof of Lemma 5.1 is completely analogous to that of Lemma 3.1, we will omit it.

Lemma 5.2. Let $U$ denote a Banach space with a normalized unconditional basis $\left(u_{j}\right)_{j=1}^{\infty}$, such that their normalized associated coordinate functionals $\left(u_{j}^{*}\right)_{j=1}^{\infty}$ form an unconditional weak ${ }^{*}$ Schauder basis of $U^{*}$. Let $U^{*}$ satisfy condition (C), $1 \leq$ $p \leq \infty$, and let $\Lambda_{i} \subset \mathbb{N}, i \in \mathbb{N}$ denote infinite sets. Let $\varphi \in\left(\ell^{p}\left(U^{*}\right)\right)^{*}$ and $\eta>0$. Then there exist infinite sets and $\mathcal{J}_{i} \subset \Lambda_{i}, i \in \mathbb{N}$ such that

$$
\sup _{\|y\|_{\ell^{p}\left(U^{*}\right) \leq 1}}\left|\left\langle\varphi, R_{\{i\} \times \mathcal{J}_{i}} y\right\rangle\right| \leq \eta, \quad i \in \mathbb{N} .
$$

Proof. If we assume Lemma 5.2 is false, then there exists an $i_{0} \in \mathbb{N}$ such that

$$
\sup _{\|y\|_{\ell p}\left(U^{*}\right) \leq 1}\left|\left\langle\varphi, R_{\left\{i_{0}\right\} \times \mathcal{A}} y\right\rangle\right|>\eta
$$

for every infinite set $\mathcal{A} \subset \Lambda_{i_{0}}$. We define $\theta=\frac{\eta}{2\|\varphi\|_{\left(\ell^{p}\left(U^{*}\right)\right)^{*} K_{u}}}$ and choose a sequence $\left(\mathcal{A}_{j}\right)_{j=1}^{\infty}$ of pairwise disjoint, infinite subsets of $\mathcal{J}$ according to $(\mathrm{C})$. By (5.4), we can find a sequence $\left(y_{j}\right)_{j=1}^{\infty}$ in $\ell^{p}\left(U^{*}\right)$ with $\left\|y_{j}\right\| \leq 1, j \in \mathbb{N}$ such that

$$
\left\langle\varphi, R_{\left\{i_{0}\right\} \times \mathcal{A}_{j}} y_{j}\right\rangle>\eta, \quad j \in \mathbb{N} .
$$

Next, define $u_{j}^{*}=q_{i_{0}} y_{j}, j \in \mathbb{N}$ and choose $\left(a_{j}\right)_{j=1}^{\infty} \in \ell^{1}$ with $\left\|\left(a_{j}\right)_{j=1}^{\infty}\right\|_{\ell^{1}}=1$ according to $(\mathrm{C})$ such that

$$
\left\|\sum_{j=1}^{\infty} a_{j} P_{\mathcal{A}_{j}} u_{j}^{*}\right\|_{U^{*}} \leq \theta=\frac{\eta}{2\|\varphi\|_{\left(\ell^{p}\left(U^{*}\right)\right)^{*}} K_{u}} .
$$


By (5.5), we obtain an integer $n \in \mathbb{N}$ such that

$$
\eta<\left\langle\varphi, \sum_{j=1}^{n} a_{j} R_{\left\{i_{0}\right\} \times \mathcal{A}_{j}} y_{j}\right\rangle \leq\|\varphi\|_{\left(\ell^{p}\left(U^{*}\right)\right)^{*}}\left\|\sum_{j=1}^{n} a_{j} R_{\left\{i_{0}\right\} \times \mathcal{A}_{j}} y_{j}\right\|_{\ell^{p}\left(U^{*}\right)} .
$$

Observe that $\sum_{j=1}^{n} a_{j} R_{\left\{i_{0}\right\} \times \mathcal{A}_{j}} y_{j}=I_{i_{0}} \sum_{j=1}^{n} a_{j} P_{\mathcal{A}_{j}} u_{j}$, hence, combining (5.6) and (5.7) leads to the contradiction

$$
\eta \leq\|\varphi\|_{\left(\ell^{p}\left(U^{*}\right)\right)^{*}}\left\|\sum_{j=1}^{n} a_{j} P_{\mathcal{A}_{j}} u_{j}\right\|_{U^{*}} \leq \eta / 2 .
$$

5.3. Proof of main result Theorem 1.2. In this section we prove our second main result Theorem 1.2, which we repeat here below (see Theorem 5.3). The proof involves inductively constructing a block basis in each coordinate of $\ell^{p}\left(S^{*}\right)$, $1 \leq p \leq \infty$. The order by which we proceed is determined by the linear order $\prec$ on $\mathbb{N}^{2}$, which is defined as follows: Let $<_{\ell}$ denote the lexicographic order on $\mathbb{N}^{2}$ and define

$$
\left(i_{0}, j_{0}\right) \prec\left(i_{1}, j_{1}\right) \quad \text { if and only if }\left(i_{0}+j_{0}, i_{0}\right)<_{\ell}\left(i_{1}+j_{1}, i_{1}\right),
$$

for all $\left(i_{0}, j_{0}\right),\left(i_{1}, j_{1}\right) \in \mathbb{N}^{2}$ (see Figure 1 ). Let $\mathcal{O}_{\prec}: \mathbb{N}^{2} \rightarrow \mathbb{N}$ denote the unique bijective function that preserves the order $\prec$, i.e.

$$
\mathcal{O}_{\prec}\left(\left(i_{0}, j_{0}\right)\right)<\mathcal{O}_{\prec}\left(\left(i_{1}, j_{1}\right)\right) \quad \text { if and only if } \quad\left(i_{0}, j_{0}\right) \prec\left(i_{1}, j_{1}\right) \text {, }
$$

for all $\left(i_{0}, j_{0}\right),\left(i_{1}, j_{1}\right) \in \mathbb{N}^{2}$.

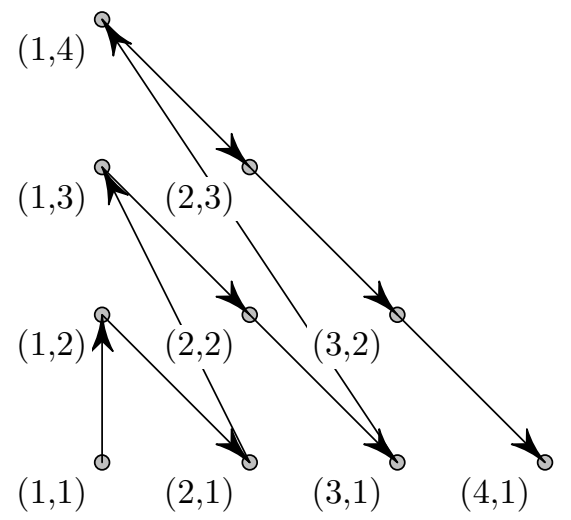

Figure 1. The first 10 Elements of $\mathbb{N}^{2}$ with respect to the order $\prec$.

Theorem 5.3 (Main result Theorem 1.2). Let $S^{*}$ denote a Banach space with a subsymmetric weak* Schauder basis satisfying condition (C). Then for all $1 \leq$ $p \leq \infty$ and any given bounded operator $T: \ell^{p}\left(S^{*}\right) \rightarrow \ell^{p}\left(S^{*}\right)$, there exist operators $M, N: \ell^{p}\left(S^{*}\right) \rightarrow \ell^{p}\left(S^{*}\right)$ such that for $H=T$ or $H=\operatorname{Id}_{\ell^{p}\left(S^{*}\right)}-T$, the diagram

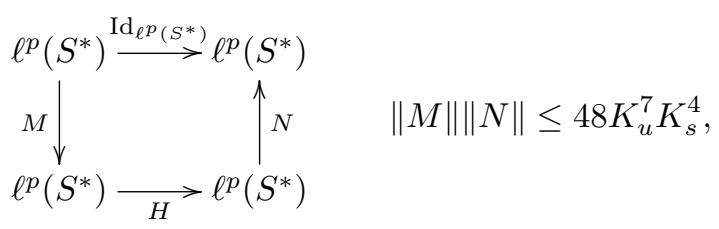

is commutative. Consequently, $\ell^{p}\left(S^{*}\right)$ is primary. 
Proof. For the definition of $e_{i j}$ and $f_{i j}$ we refer to Section 5.1. Within this proof, we identify

$$
\eta_{k} \leftrightarrow \eta_{k_{0} k_{1}}, \quad \mathcal{B}_{k} \leftrightarrow \mathcal{B}_{k_{0} k_{1}}, \quad b_{k} \leftrightarrow b_{k_{0} k_{1}}, \quad b_{k}^{*} \leftrightarrow b_{k_{0} k_{1}}^{*},
$$

whenever $\mathcal{O}_{\prec}\left(\left(k_{0}, k_{1}\right)\right)=k$. We conclude this preliminary step by defining

$$
\eta_{k}=K_{u}^{-1} 4^{-k-1}, \quad k \in \mathbb{N} \text {. }
$$

Step 1: Inductive COnstruction. We will now inductively (with respect to the order $\prec$ on $\mathbb{N}^{2}$ ) construct sequences $\left(b_{i j}^{(\varepsilon)}\right)_{i, j=1}^{\infty}$ and $\left(b_{i j}^{*(\varepsilon)}\right)_{i, j=1}^{\infty}$. We begin by putting $\mathcal{J}_{1, i}=\mathbb{N}, i \in \mathbb{N}, \mathcal{B}_{1}=\{(1,1),(1,2)\}$ and define

$$
b_{11}^{(\varepsilon)}=e_{11}-e_{12} \quad \text { and } \quad b_{11}^{*(\varepsilon)}=f_{11}-f_{12} .
$$

By Lemma 5.2, there exist infinite sets $\mathcal{J}_{2, i} \subset \mathbb{N}, i \in \mathbb{N}$ with

$$
\partial_{2, i} \subset \mathcal{J}_{1, i} \backslash\{1,2\},
$$

such that

$$
\sup _{\|y\|_{\ell p}\left(S^{*}\right) \leq 1}\left|\left\langle T^{*} b_{1}^{*(\varepsilon)}, R_{\{i\} \times \mathcal{J}_{2, i}} y\right\rangle\right| \leq \eta_{1}, \quad i \in \mathbb{N} .
$$

Let us assume that we have already

$\triangleright$ selected infinite sets $\mathcal{J}_{k, i} \subset \mathbb{N}, i \in \mathbb{N}, 1 \leq k \leq K$ and finite sets $\mathcal{B}_{k} \subset \mathbb{N}^{2}$ with $\left|\mathcal{B}_{k}\right|=2,1 \leq k \leq K-1$ such that

$$
\mathcal{B}_{k}=\mathcal{B}_{k_{0} k_{1}} \subset\left(\left\{k_{0}\right\} \times \mathcal{J}_{k, k_{0}}\right) \backslash\left(\left\{k_{0}\right\} \times \mathcal{J}_{k+1, k_{0}}\right),
$$

whenever $1 \leq k \leq K-1$ and $\mathcal{O}_{\prec}\left(\left(k_{0}, k_{1}\right)\right)=k$, and that

$$
\mathcal{J}_{k+1, i} \subset \mathcal{J}_{k, i} \backslash\left\{j \in \mathbb{N}: j \leq \max \left\{j^{\prime} \in \mathbb{N}: \exists i^{\prime} \in \mathbb{N},\left(i^{\prime}, j^{\prime}\right) \in \bigcup_{\ell=1}^{k} \mathcal{B}_{\ell}\right\}\right\},
$$

for all $i \in \mathbb{N}$ and $1 \leq k \leq K-1$;

$\triangleright$ defined $b_{k}^{(\varepsilon)}, b_{k}^{*(\varepsilon)}$ by

$$
b_{k}^{(\varepsilon)}=e_{k_{0} j}-e_{k_{0} j^{\prime}} \quad \text { and } \quad b_{k}^{*(\varepsilon)}=f_{k_{0} j}-f_{k_{0} j^{\prime}},
$$

whenever $1 \leq k \leq K-1, \mathcal{O}_{\prec}\left(\left(k_{0}, k_{1}\right)\right)=k$ and $\left(k_{0}, j\right),\left(k_{0}, j^{\prime}\right) \in \mathcal{B}_{k}=\mathcal{B}_{k_{0} k_{1}}$ with $j<j^{\prime}$;

$\triangleright$ obtained the estimates

$$
\begin{aligned}
& \qquad \sum_{\ell=1}^{k-1}\left|\left\langle b_{k}^{*(\varepsilon)}, T b_{\ell}^{(\varepsilon)}\right\rangle\right| \leq \eta_{k}, \\
& \sup _{\|y\|_{\ell}\left(^{*}\right) \leq 1}\left|\left\langle T^{*} b_{k}^{*(\varepsilon)}, R_{\{i\} \times \mathcal{J}_{k+1, i}} y\right\rangle\right| \leq \eta_{k}, \\
& \text { for all } 1 \leq k \leq K-1 .
\end{aligned}
$$

We now come to the inductive step. To this end, choose $\left(K_{0}, K_{1}\right) \in \mathbb{N}^{2}$ with $\mathcal{O}_{\prec}\left(\left(K_{0}, K_{1}\right)\right)=K$. The set $\mathcal{J}_{K, K_{0}}$ is infinite (see $\left.(5.14 \mathrm{a})\right)$, hence, by Lemma 5.1 there exists a set $\mathcal{F} \subset \mathcal{J}_{K, K_{0}}$ with $|\mathcal{F}|=2$ such that

$$
\sum_{\ell=1}^{K-1}\left|\left\langle f_{K_{0} j}-f_{K_{0} j^{\prime}}, T b_{\ell}^{(\varepsilon)}\right\rangle\right| \leq \eta_{K}, \quad j, j^{\prime} \in \mathcal{F} .
$$

We define the collection

$$
\mathcal{B}_{K}=\mathcal{B}_{K_{0} K_{1}}=\left\{K_{0}\right\} \times \mathcal{F},
$$

and note that (with the possible exception of $\left.\mathcal{B}_{K} \cap\left(\left\{K_{0}\right\} \times \mathcal{J}_{K+1, K_{0}}\right)=\emptyset\right)$ condition (5.14a) holds true for $K+1$. We will take care of the possible exception by 
appropriately choosing $\mathcal{J}_{K+1, i}, i \in \mathbb{N}$ at a later stage of the proof. Next, we define the functions $b_{K}^{(\varepsilon)}, b_{K}^{*(\varepsilon)}$ and the signs $\varepsilon_{K_{0}, j}, j \in \mathcal{F}$ by

$$
b_{K}^{(\varepsilon)}=e_{K_{0} j}-e_{K_{0} j^{\prime}}, \quad b_{K}^{*(\varepsilon)}=f_{K_{0} j}-f_{K_{0} j^{\prime}}, \quad j, j^{\prime} \in \mathcal{F}, j<j^{\prime},
$$

which is in accordance with (5.14c). Combining (5.15) with (5.18) yields

$$
\sum_{\ell=1}^{K-1}\left|\left\langle b_{K}^{*(\varepsilon)}, T b_{\ell}^{(\varepsilon)}\right\rangle\right| \leq \eta_{K}
$$

showing that (5.14d) holds for $K+1$, as required. Since all the sets $\mathcal{B}_{k}, 1 \leq k \leq K$ are finite, we can apply Lemma 5.2 to find infinite sets $\mathcal{J}_{K+1, i}, i \in \mathbb{N}$ with

$$
\mathcal{J}_{K+1, i} \subset \mathcal{J}_{K, i} \backslash\left\{j \in \mathbb{N}: j \leq \max \left\{j^{\prime} \in \mathbb{N}: \exists i^{\prime} \in \mathbb{N},\left(i^{\prime}, j^{\prime}\right) \in \bigcup_{\ell=1}^{K} \mathcal{B}_{\ell}\right\}\right\},
$$

such that the following estimate is satisfied:

$$
\sup _{\|y\|_{\ell p}\left(S^{*}\right) \leq 1}\left|\left\langle T^{*} b_{K}^{*(\varepsilon)}, R_{\{i\} \times \mathcal{J}_{K+1, i}} y\right\rangle\right| \leq \eta_{K}, \quad i \in \mathbb{N},
$$

Note that (5.19) and (5.20) imply (5.14b) and (5.14e) for $K+1$. Moreover, (5.19) also implies $\mathcal{B}_{K} \cap\left(\left\{K_{0}\right\} \times \mathcal{J}_{K+1, K_{0}}\right)=\emptyset$, which completes the missing part of (5.14a) for $K+1$ (see (5.16)).

Altogether, we completed the inductive step, and we know that (5.14) is true for $K+1$.

Step 2: Basic operators. We define the operators $B, Q: \ell^{p}\left(S^{*}\right) \rightarrow \ell^{p}\left(S^{*}\right)$ by

$$
\begin{aligned}
& B y=\sum_{i, j=1}^{\infty}\left\langle f_{i j}, y\right\rangle \frac{1}{\left\|b_{i j}^{(\varepsilon)}\right\|_{\ell^{p}\left(S^{*}\right)}} b_{i j}^{(\varepsilon)}, \quad y \in \ell^{p}\left(S^{*}\right), \\
& Q y=\sum_{i, j=1}^{\infty} \frac{\left\|b_{i j}^{(\varepsilon)}\right\|_{\ell^{p}\left(S^{*}\right)}}{\left|\mathcal{B}_{i j}\right|}\left\langle b_{i j}^{*(\varepsilon)}, y\right\rangle e_{i j}, \quad y \in \ell^{p}\left(S^{*}\right) .
\end{aligned}
$$

For the mode of convergence of the above series, we refer to (5.1).

We will now show that $B$ and $Q$ are bounded linear operators. To this end, let $y \in \ell^{p}\left(S^{*}\right)$ and observe that by $(5.14 \mathrm{c}),\|B y\|_{\ell^{p}\left(S^{*}\right)}$ is dominated by

$$
\left\|\sum_{i, j=1}^{\infty}\left\langle f_{i j}, y\right\rangle \frac{1}{\left\|b_{i j}^{(\varepsilon)}\right\|_{\ell^{p}\left(S^{*}\right)}} e_{k_{i j} \ell_{i j}}\right\|_{\ell^{p}\left(S^{*}\right)}+\left\|\sum_{i, j=1}^{\infty}\left\langle f_{i j}, y\right\rangle \frac{1}{\left\|b_{i j}^{(\varepsilon)}\right\|_{\ell^{p}\left(S^{*}\right)}} e_{k_{i j} m_{i j}}\right\|_{\ell^{p}\left(S^{*}\right)},
$$

where $\left\{\left(k_{i j}, \ell_{i j}\right),\left(k_{i j}, m_{i j}\right)\right\}=\mathcal{B}_{i j}, i, j \in \mathbb{N}$. By unconditionality we obtain that $\left\|b_{i j}^{(\varepsilon)}\right\|_{\ell^{p}\left(S^{*}\right)} \geq K_{u}^{-1}, i, j \in \mathbb{N}$, and subsequently

$$
\|B y\|_{\ell^{p}\left(S^{*}\right)} \leq K_{u}^{2}\left\|\sum_{i, j=1}^{\infty}\left\langle f_{i j}, y\right\rangle e_{k_{i j} \ell_{i j}}\right\|_{\ell^{p}\left(S^{*}\right)}+K_{u}^{2}\left\|\sum_{i, j=1}^{\infty}\left\langle f_{i j}, y\right\rangle e_{k_{i j} m_{i j}}\right\|_{\ell^{p}\left(S^{*}\right)} .
$$

Furthermore, by (5.14b) we have that $\ell_{i j}<\ell_{i(j+1)}$ and $m_{i j}<m_{i(j+1)}, i, j \in \mathbb{N}$. The weak* Schauder basis $\left(s_{j}^{*}\right)_{j=1}^{\infty}$ of $S^{*}$ is subsymmetric, hence

$$
\begin{aligned}
\|B y\|_{\ell^{p}\left(S^{*}\right)} & \leq 2 K_{u}^{2} K_{s}\left\|\sum_{i, j=1}^{\infty}\left\langle f_{i j}, y\right\rangle e_{k_{i j} j}\right\|_{\ell^{p}\left(S^{*}\right)}=2 K_{u}^{2} K_{s}\left\|\sum_{i, j=1}^{\infty}\left\langle f_{i j}, y\right\rangle e_{i j}\right\|_{\ell^{p}\left(S^{*}\right)} \\
& =2 K_{u}^{2} K_{s}\|y\|_{\ell^{p}\left(S^{*}\right)} .
\end{aligned}
$$


Similarly, we obtain that $\|Q y\|_{\ell^{p}\left(S^{*}\right)}$ is dominated by

$$
\left\|\sum_{i, j=1}^{\infty} \frac{\left\|b_{i j}^{(\varepsilon)}\right\|_{\ell^{p}\left(S^{*}\right)}}{\left|\mathcal{B}_{i j}\right|}\left\langle f_{k_{i j} \ell_{i j}}, y\right\rangle e_{i j}\right\|_{\ell^{p}\left(S^{*}\right)}+\left\|\sum_{i, j=1}^{\infty} \frac{\left\|b_{i j}^{(\varepsilon)}\right\|_{\ell^{p}\left(S^{*}\right)}}{\left|\mathcal{B}_{i j}\right|}\left\langle f_{k_{i j} m_{i j}}, y\right\rangle e_{i j}\right\|_{\ell^{p}\left(S^{*}\right)}
$$

which, since $\left(s_{j}^{*}\right)_{j=1}^{\infty}$ is subsymmetric, gives us the following upper bound for $\|Q y\|_{\ell^{p}\left(S^{*}\right)}$ : $K_{s}\left\|\sum_{i, j=1}^{\infty} \frac{\left\|b_{i j}^{(\varepsilon)}\right\|_{\ell^{p}\left(S^{*}\right)}}{\left|\mathcal{B}_{i j}\right|}\left\langle f_{k_{i j} \ell_{i j}}, y\right\rangle e_{i \ell_{i j}}\right\|_{\ell^{p}\left(S^{*}\right)}+K_{s}\left\|\sum_{i, j=1}^{\infty} \frac{\left\|b_{i j}^{(\varepsilon)}\right\|_{\ell^{p}\left(S^{*}\right)}}{\left|\mathcal{B}_{i j}\right|}\left\langle f_{k_{i j} m_{i j}}, y\right\rangle e_{i m_{i j}}\right\|_{\ell^{p}\left(S^{*}\right)}$.

Using $\frac{\left\|b_{i j}^{(\varepsilon)}\right\|_{\ell p}\left(S^{*}\right)}{\left|\mathcal{B}_{i j}\right|} \leq 1$, we obtain by unconditionality

$$
\|Q y\|_{\ell^{p}\left(S^{*}\right)} \leq 2 K_{s} K_{u}\|y\|_{\ell^{p}\left(S^{*}\right)} .
$$

One can easily verify that $Q B=\operatorname{Id}_{\ell^{p}\left(S^{*}\right)}$, i.e. the diagram

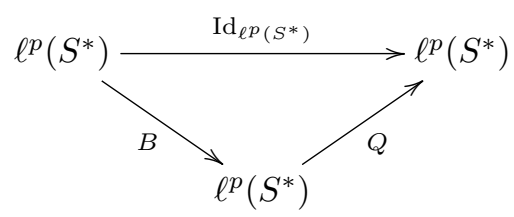

is commutative. Consequently, $B$ is an isomorphism onto its range, and its range is complemented by $B Q$.

Step 3: Factorization of the IDentity. With $i \in \mathbb{N}$ fixed, observe that at least one of the two following sets is infinite:

$\mathcal{K}_{i}=\left\{j \in \mathbb{N}:\left|\left\langle b_{i j}^{*(\varepsilon)}, T b_{i j}^{(\varepsilon)}\right\rangle\right| \geq 1\right\}, \quad \mathcal{L}_{i}=\left\{j \in \mathbb{N}:\left|\left\langle b_{i j}^{*(\varepsilon)},\left(\operatorname{Id}_{\ell^{p}\left(S^{*}\right)}-T\right) b_{i j}^{(\varepsilon)}\right\rangle\right| \geq 1\right\}$.

Thus, clearly one of the following two sets is infinite:

$$
\left\{i \in \mathbb{N}: \mathcal{K}_{i} \text { is infinite }\right\} \quad \text { or } \quad\left\{i \in \mathbb{N}: \mathcal{L}_{i} \text { is infinite }\right\}
$$

If the left set is infinite, we denote it by $\mathcal{J}$ and we put $\mathcal{J}_{i}=\mathcal{K}_{i}, i \in \mathcal{J}$ as well as $H=T$; if the left set is finite, we denote the right set by $\mathcal{J}$, and we define $\mathcal{J}_{i}=\mathcal{L}_{i}$, $i \in \mathcal{J}$ as well as $H=\operatorname{Id}_{\ell^{p}\left(S^{*}\right)}-T$. In either of these two cases, we obtain that

$\mathcal{J}$ is infinite, $\mathcal{J}_{i}$ is infinite, $i \in \mathcal{J}$ and $\left|\left\langle b_{i j}^{*(\varepsilon)}, H b_{i j}^{(\varepsilon)}\right\rangle\right| \geq 1, i \in \mathcal{J}, j \in \mathcal{J}_{i}$.

Let $Y=B\left(\ell^{p}\left(S^{*}\right)\right)$ and define $A: \ell^{p}\left(S^{*}\right) \rightarrow Y, y \mapsto B y$. Thus, by (5.22), (5.23) and (5.24), the following diagram is commutative:

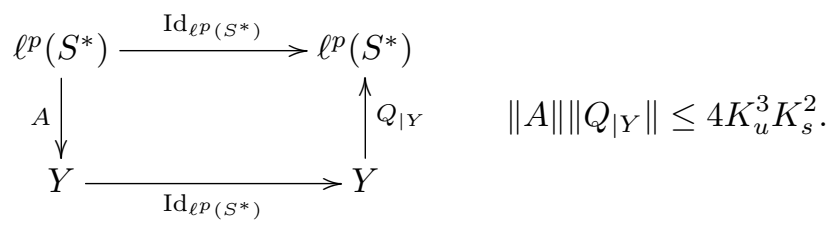

Now, put $\mathcal{K}=\left\{\mathcal{O}_{\prec}(i, j): i \in \mathcal{J}, j \in \mathcal{J}_{i}\right\}$ and define $P: \ell^{p}\left(S^{*}\right) \rightarrow Y$ by

$$
P y=\sum_{k \in \mathcal{K}} \frac{\left\langle b_{k}^{*(\varepsilon)}, y\right\rangle}{\left\langle b_{k}^{*(\varepsilon)}, H b_{k}^{(\varepsilon)}\right\rangle} b_{k}^{(\varepsilon)}, \quad y \in \ell^{p}\left(S^{*}\right) .
$$

Recall that at the beginning of the proof we identified $b_{k}^{(\varepsilon)}$ with $b_{k_{0} k_{1}}^{(\varepsilon)}$ and $b_{k}^{*(\varepsilon)}$ with $b_{k_{0} k_{1}}^{*(\varepsilon)}$, whenever $\mathcal{O}_{\prec}\left(\left(k_{0}, k_{1}\right)\right)=k$. Observe that by $(5.25)$, unconditionality and the definition of $B$ and $Q$ (see (5.21)), we obtain that

$$
\|P y\|_{\ell^{p}\left(S^{*}\right)} \leq 2 K_{u}\|B Q y\|_{\ell^{p}\left(S^{*}\right)}
$$


SUBSYMMETRIC WEAK* SCHAUDER BASES AND FACTORIZATION OF THE IDENTITY15

Combining the latter estimate with (5.22) and (5.23) yields

$$
\|P y\|_{\ell^{p}\left(S^{*}\right)} \leq 8 K_{u}^{4} K_{s}^{2}\|y\|_{\ell^{p}\left(S^{*}\right)} .
$$

A straightforward calculation shows that for all $y=\sum_{k \in \mathcal{K}} a_{k} b_{k}^{(\varepsilon)} \in Y$, the following identity is true:

$$
P H y-y=\sum_{k \in \mathcal{K}} \sum_{\ell \in \mathcal{K}: \ell<k} a_{\ell} \frac{\left\langle b_{k}^{*(\varepsilon)}, H b_{\ell}^{(\varepsilon)}\right\rangle}{\left\langle b_{k}^{*(\varepsilon)}, H b_{k}^{(\varepsilon)}\right\rangle} b_{k}^{(\varepsilon)}+\sum_{k \in \mathcal{K}} \frac{\left\langle H^{*} b_{k}^{*(\varepsilon)}, \sum_{\ell \in \mathcal{K}: \ell>k} a_{\ell} b_{\ell}^{(\varepsilon)}\right\rangle}{\left\langle b_{k}^{*(\varepsilon)}, H b_{k}^{(\varepsilon)}\right\rangle} b_{k}^{(\varepsilon)} .
$$

Since $\left|\left\langle b_{j}^{(\varepsilon)}, y\right\rangle\right| \leq\left\|b_{j}^{(\varepsilon)}\right\|_{S}\|y\|_{\ell^{p}\left(S^{*}\right)}$, we obtain $\left|a_{j}\right| \leq\|y\|_{\ell^{p}\left(S^{*}\right)}$. Hence, by (5.25) and the off-diagonal estimate $(5.14 \mathrm{~d})$, we obtain

$$
\left\|\sum_{k \in \mathcal{K}} \sum_{\ell \in \mathcal{K}: \ell<k} a_{\ell} \frac{\left\langle b_{k}^{*(\varepsilon)}, H b_{\ell}^{(\varepsilon)}\right\rangle}{\left\langle b_{k}^{*(\varepsilon)}, H b_{k}^{(\varepsilon)}\right\rangle} b_{k}^{(\varepsilon)}\right\|_{\ell^{p}\left(S^{*}\right)} \leq 2\|y\|_{\ell^{p}\left(S^{*}\right)} \sum_{k \in \mathbb{N}} \eta_{k} .
$$

Using $(5.25),(5.14 \mathrm{~b}),(5.14 \mathrm{a})$ and the other off-diagonal estimate (5.14e) yields

$$
\left\|\sum_{k \in \mathcal{K}} \frac{\left\langle H^{*} b_{k}^{*(\varepsilon)}, \sum_{\ell \in \mathcal{K}: \ell>k} a_{\ell} b_{\ell}^{(\varepsilon)}\right\rangle}{\left\langle b_{k}^{*(\varepsilon)}, H b_{k}^{(\varepsilon)}\right\rangle} b_{k}^{(\varepsilon)}\right\|_{\ell^{p}\left(S^{*}\right)} \leq 2 \sum_{k \in \mathcal{K}} \eta_{k}\left\|\sum_{\ell \in \mathcal{K}: \ell>k} a_{\ell} b_{\ell}^{(\varepsilon)}\right\|_{\ell^{p}\left(S^{*}\right)} .
$$

By (5.21a), estimate (5.22) and by unconditionality, we obtain

$$
\left\|\sum_{\ell \in \mathcal{K}: \ell>k} a_{\ell} b_{\ell}^{(\varepsilon)}\right\|_{\ell^{p}\left(S^{*}\right)} \leq 2 K_{u}^{3} K_{s}\|y\|_{\ell^{p}\left(S^{*}\right)}
$$

The latter estimate together with (5.31) yields

$$
\left\|\sum_{k \in \mathcal{K}} \frac{\left\langle H^{*} b_{k}^{*(\varepsilon)}, \sum_{\ell \in \mathcal{K}: \ell>k} a_{\ell} b_{\ell}^{(\varepsilon)}\right\rangle}{\left\langle b_{k}^{*(\varepsilon)}, H b_{k}^{(\varepsilon)}\right\rangle} b_{k}^{(\varepsilon)}\right\|_{\ell^{p}\left(S^{*}\right)} \leq 4 K_{u}^{3} K_{s} \sum_{k \in \mathcal{K}} \eta_{k}\|y\|_{\ell^{p}\left(S^{*}\right)}
$$

Recall that we put $\eta_{k}=K_{u}^{-1} 4^{-k-1}, k \in \mathbb{N}$, (see (5.10)). Combining the estimates (5.30) and (5.32) with (5.29) yields

$$
\|P H y-y\|_{\ell^{p}\left(S^{*}\right)} \leq \frac{1}{3}\|y\|_{\ell^{p}\left(S^{*}\right)}, \quad y \in Y .
$$

Let $J: Y \rightarrow \ell^{p}\left(S^{*}\right)$ denote the operator given by $J y=y$, then PHJ $Y \rightarrow Y$ is invertible by (5.33). Thus, if we define $V=(P H J)^{-1} P$, the following diagram is commutative:

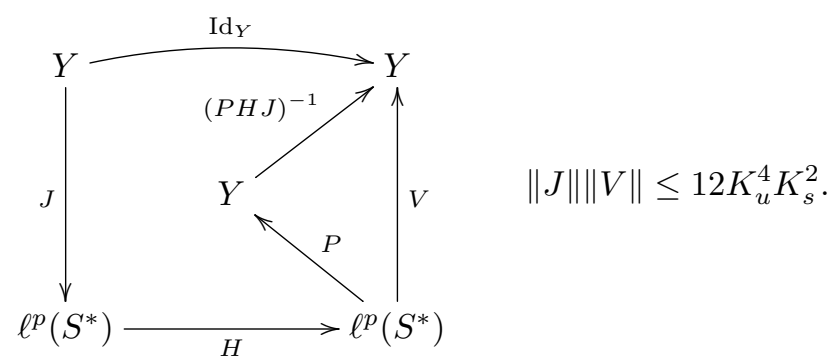


The operators $J$ and $V$ are both bounded, thus merging the diagrams (5.26) and (5.34) yields the commutative diagram

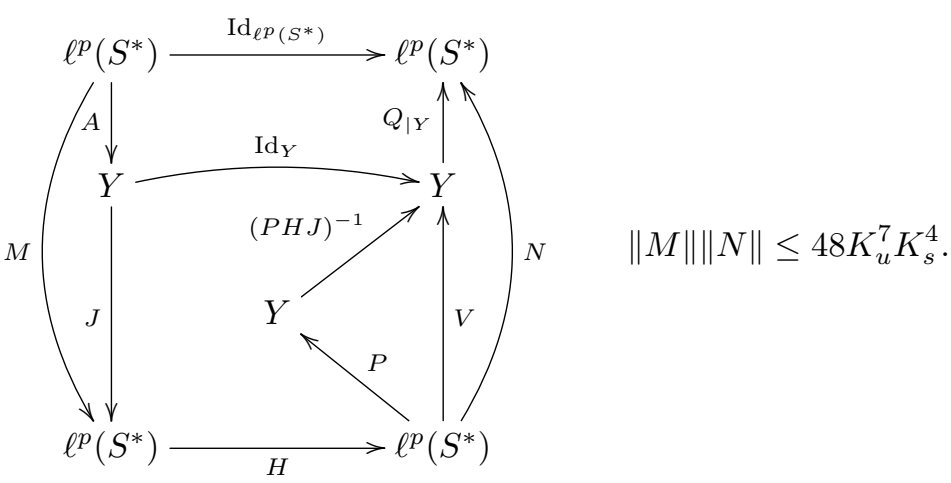

STEP 4: $\ell^{p}\left(S^{*}\right)$ IS PRIMARY. Let $Q: \ell^{p}\left(S^{*}\right) \rightarrow \ell^{p}\left(S^{*}\right)$ denote a bounded projection. Then by (5.9), we know that $\ell^{p}\left(S^{*}\right)$ is either isomorphic to a complemented subspace of $Q\left(\ell^{p}\left(S^{*}\right)\right)$, or isomorphic to a complemented subspace of $\left(\operatorname{Id}_{\ell^{p}\left(S^{*}\right)}-Q\right)\left(\ell^{p}\left(S^{*}\right)\right)$. Moreover, since $\ell^{p}\left(S^{*}\right)$ is the $\ell^{p}$-sum of a Banach space, $\ell^{p}\left(S^{*}\right)$ is isomorphic to $\ell^{p}\left(\ell^{p}\left(S^{*}\right)\right)$. Thus, by Pełczyński's decomposition method (see [7]; see also [9, II.B.24]), we obtain that either $Q\left(\ell^{p}\left(S^{*}\right)\right)$ is isomorphic to $\ell^{p}\left(S^{*}\right)$, or $\left(\operatorname{Id}_{\ell^{p}\left(S^{*}\right)}-Q\right)\left(\ell^{p}\left(S^{*}\right)\right)$ is isomorphic to $\ell^{p}\left(S^{*}\right)$.

\section{Acknowledgments.}

It is my pleasure to thank P.F.X. Müller for many helpful discussions. Supported by the Austrian Science Foundation (FWF) Pr.Nr. P28352.

\section{REFERENCES}

[1] M. Capon. Primarité de certains espaces de Banach. Proc. London Math. Soc. (3), 45(1):113$130,1982$.

[2] P. G. Casazza, C. A. Kottman, and B. L. Lin. On some classes of primary Banach spaces. Canad. J. Math., 29(4):856-873, 1977.

[3] P. G. Casazza and B. L. Lin. Projections on Banach spaces with symmetric bases. Studia Math., 52:189-193, 1974.

[4] R. Lechner. Factorization in $S L^{\infty}$. Israel J. Math., to appear. Preprint available on ArXiv https://arxiv.org/abs/1611.00622.

[5] J. Lindenstrauss. On complemented subspaces of m. Israel J. Math., 5:153-156, 1967.

[6] J. Lindenstrauss and L. Tzafriri. Classical Banach spaces. I. Springer-Verlag, Berlin-New York, 1977. Sequence spaces, Ergebnisse der Mathematik und ihrer Grenzgebiete, Vol. 92.

[7] A. Pełczyński. Projections in certain Banach spaces. Studia Math., 19:209-228, 1960.

[8] I. Singer. Bases in Banach spaces. I. Springer-Verlag, New York-Berlin, 1970. Die Grundlehren der mathematischen Wissenschaften, Band 154.

[9] P. Wojtaszczyk. Banach spaces for analysts, volume 25 of Cambridge Studies in Advanced Mathematics. Cambridge University Press, Cambridge, 1991.

Richard Lechner, Institute of Analysis, Johannes Kepler University Linz, Altenberger Strasse 69, A-4040 Linz, Austria

E-mail address: Richard.Lechner@jku.at 